\title{
Bronchial artery aneurysm mimicking aortic arch aneurysm or aortic dissection
}

Shinichi Ishida, MD, ${ }^{\mathrm{a}}$ Wataru Koike, MD, PhD, ${ }^{\mathrm{b}}$ Takashi Fujita, MD, ${ }^{\mathrm{a}}$ and Kei Yagami, MD, PhD, ${ }^{\mathrm{a}}$ Gifu, Japan

From the Departments of ${ }^{\mathrm{a} C a r d i a c}$ Surgery, and ${ }^{\mathrm{b}}$ Diagnostic Radiology, Gifu Prefectural Tajimi Hospital, Gifu, Japan.

Disclosures: The authors reported no conflicts of interest.

The Journal policy requires editors and reviewers to disclose conflicts of interest and to decline handling or reviewing manuscripts for which they may have a conflict of interest. The editors and reviewers of this article have no conflicts of interest.

Received for publication May 24, 2020; revisions received May 24, 2020; accepted for publication June 16, 2020; available ahead of print June 20, 2020.

Address for reprints: Shinichi Ishida, MD, 5-161 Maebata-cho, Tajimi-city, Gifu 507-8522, Japan (E-mail: shin1dinho@yahoo.co.jp).

JTCVS Techniques 2020;3:54-6

2666-2507

Copyright $(9) 2020$ The Authors. Published by Elsevier Inc. on behalf of The American Association for Thoracic Surgery. This is an open access article under the CC BY-NC-ND license (http://creativecommons.org/licenses/bync-nd/4.0/).

https://doi.org/10.1016/j.xjtc.2020.06.016

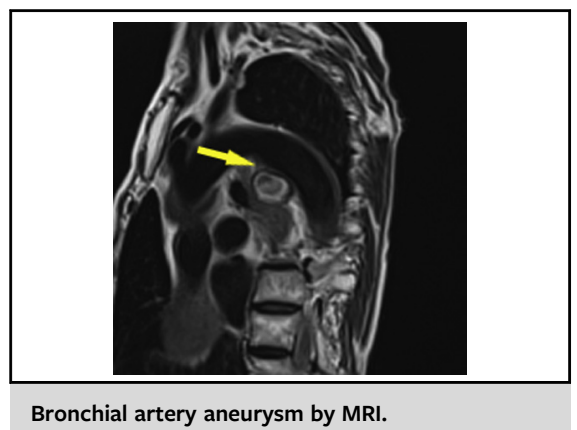

CENTRAL MESSAGE

Magnetic resonance imaging was

useful for bronchial artery aneu-

rysms that were difficult to

distinguish from an aortic arch

aneurysm or a thrombosed type

of aortic dissection via

computed tomography.

See Commentaries on pages 57 and 59.

Bronchial artery aneurysms (BAAs) are rare. They may be asymptomatic and detected incidentally; however, they can cause airway bleeding, mediastinal bleeding, and sometimes death if they rupture. Here, we present a case of a BAA that was detected secondary to chest pain. It was difficult to diagnose, but bronchial artery embolization (BAE) revealed good results.

\section{CLINICAL SUMMARY}

An 82-year-old woman presented with chest pain. An enhanced computed tomography (CT) scan that was conducted at a previous hospital suspected an impending rupture of an aortic arch aneurysm. She was therefore
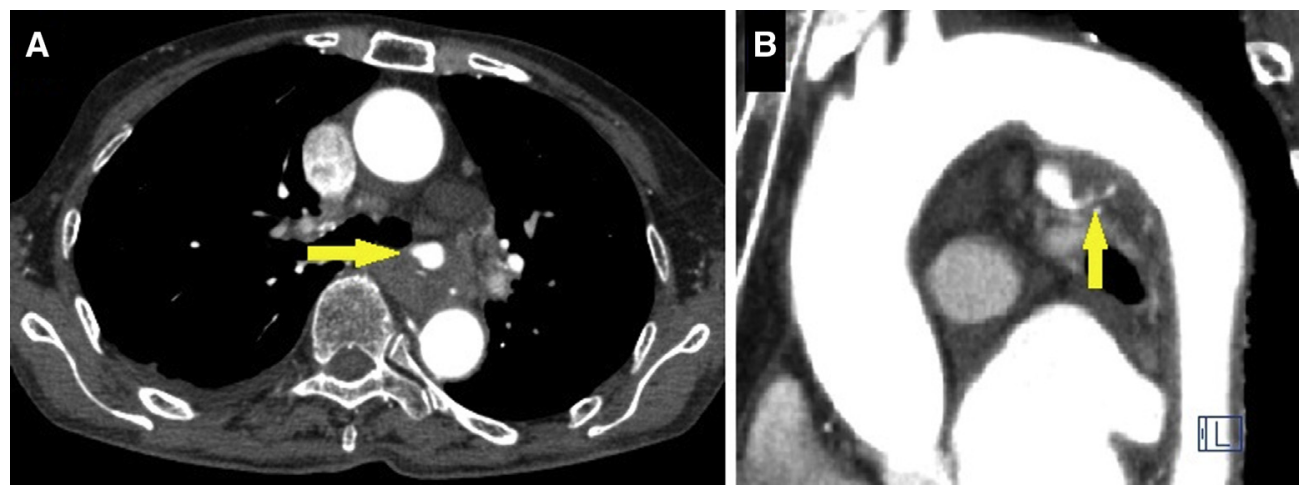

FIGURE 1. A, Enhanced computed tomography scan showing a soft tissue-shadow on the caudal side of the aortic arch, and a nodular contrast region inside (arrow). B, In a sagittal plane, the contrast region seemed to be continuous with the aortic arch, but it was unclear if the contrast region was inside the adventitia of the aorta (arrow). 


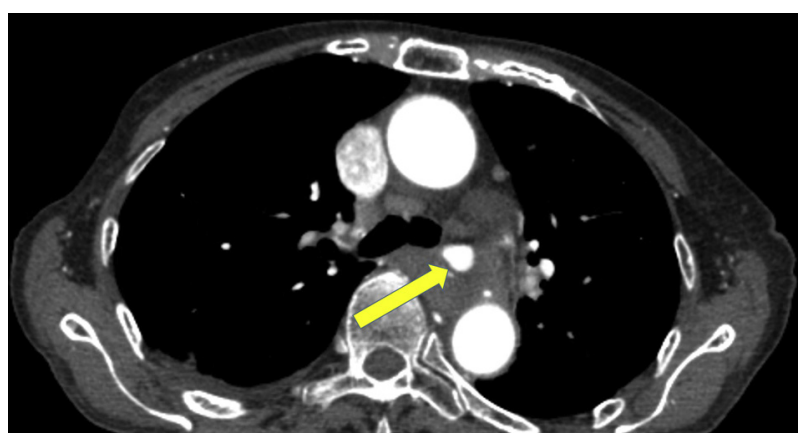

VIDEO 1. Enhanced CT at our hospital showing a soft-tissue shadow on the caudal side of the aortic arch and a nodular contrast region inside (arrow). Video available at: https://www.jtcvs.org/article/S2666-2507(20) 30294-7/fulltext.

referred for surgery. Another CT was performed at our hospital. It showed a soft-tissue shadow on the caudal side of the aortic arch, and a nodular contrast region inside (Figure 1, A; Video 1). An aortic arch aneurysm was initially suspected, but it was atypical that the nodular contrast region was not continuous with the aortic lumen. Consequently, we judged that it was likely to be intramural blood pools complicated with a thrombosed type of aortic dissection. Because the contrast region seemed to be continuous with the bronchial artery branching from the aortic arch in a sagittal plane (Figure 1, $B$ ), we considered the possibility of a hematoma or extravasation secondary to bleeding from the bronchial artery. However, because the size of the soft-tissue shadow was not markedly different from that on previous CT, active bleeding was considered unlikely. In addition, her chest pain stopped after being transported to our hospital, and no abnormality was found on the blood test, including an increase in inflammatory reaction. Hence, inflammatory diseases such as pleuritis were also negative, and the nodular contrast region on imaging was thought to be the cause of the symptoms.

We, therefore, started treatment with rest and an antihypertensive. Chest pain passed without appearance during resting treatment. A magnetic resonance imaging (MRI) scan that was conducted after 13 days showed an aneurysm of $25 \times 14 \mathrm{~mm}$ and that the adventitia of the aorta was present between the aneurysm and the aortic arch (Figure 2, A). It suggested that the aneurysm did not originate from the aorta. We performed a third CT scan, which showed that the nodular contrast region was expanding. Given the MRI findings and the fact that the aneurysm was expanding, we concluded that it was a BAA. We performed bronchial arteriography and BAE because of the tendency to worsen in image findings (Figure 2, $B$, Video 2). Embolization was performed using coils, and the disappearance of the aneurysm was confirmed by the CT scan after embolization (Videos 3 and 4). The postoperative course was uneventful. Written informed consent was obtained from the patient for publication of this case report.

\section{DISCUSSION}

BAAs are rare and are observed in less than $1 \%$ of all bronchial angiography cases. ${ }^{1}$ They are classified into intrapulmonary and mediastinal types according to the site of location. The intrapulmonary type is mostly discovered secondary to hemoptysis. In contrast, the mediastinal type is often discovered secondary to a symptom involving the esophagus or the heart, or an aortic dissection-like symptom such as chest or back pain as in this case. ${ }^{2}$
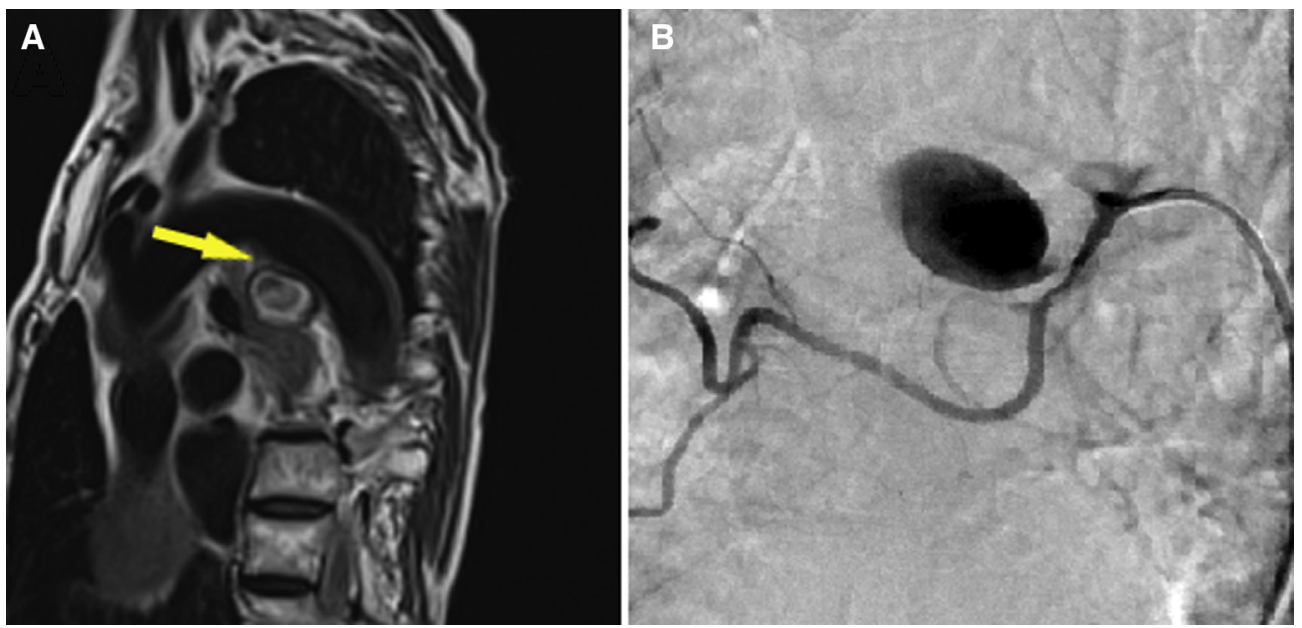

FIGURE 2. A, Magnetic resonance imaging of T1 showing that the adventitia of the aorta (arrow) was present between the aneurysm and the aortic arch. B, Bronchial artery angiography showing a bronchial artery aneurysm. 


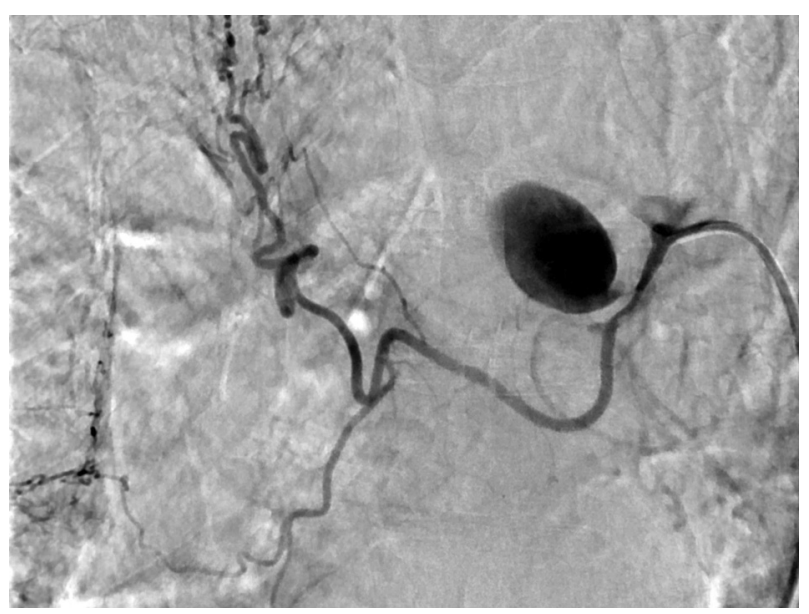

VIDEO 2. Bronchial artery angiography showing a bronchial artery aneurysm. Video available at: https://www.jtcvs.org/article/S2666-2507(20) 30294-7/fulltext.

In many cases, the diagnosis is obtained by enhanced $\mathrm{CT}^{3}$ However, in this case because it was close to the aorta, we initially determined it to be intramural blood pools associated with a thrombosed type of aortic dissection. MRI was very useful for diagnosis because it identified the presence of the adventitia of the aorta at the boundary between the aneurysm and the aortic lumen. Although MRI is frequently used to evaluate aneurysms in the field of neurosurgery, it is less commonly used in thoracic vascular surgery. It has been reported that MRI was used to diagnose a bronchial aneurysm that needed

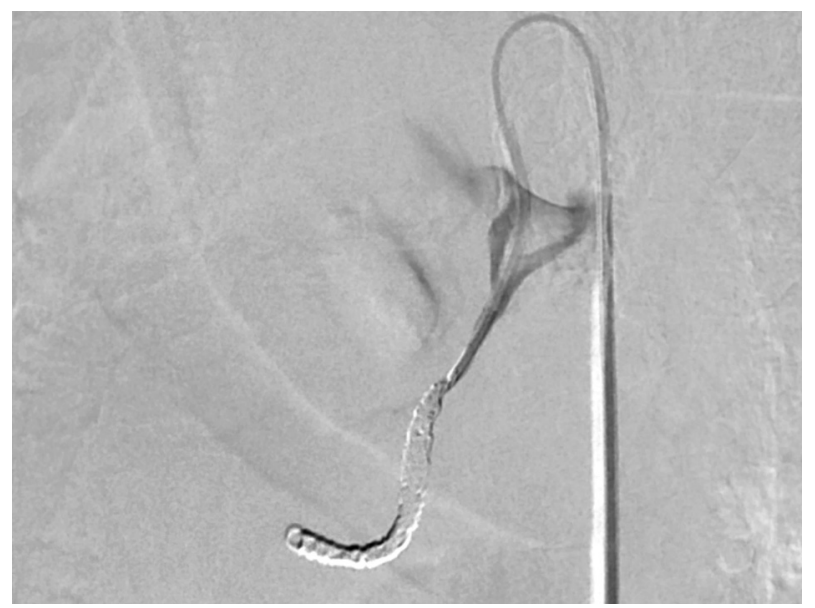

VIDEO 3. Embolization was performed using coils to embolize the bronchial artery from the distal side to the proximal side of the aneurysm. Video available at: https://www.jtcvs.org/article/S2666-2507(20)30294-7/ fulltext.

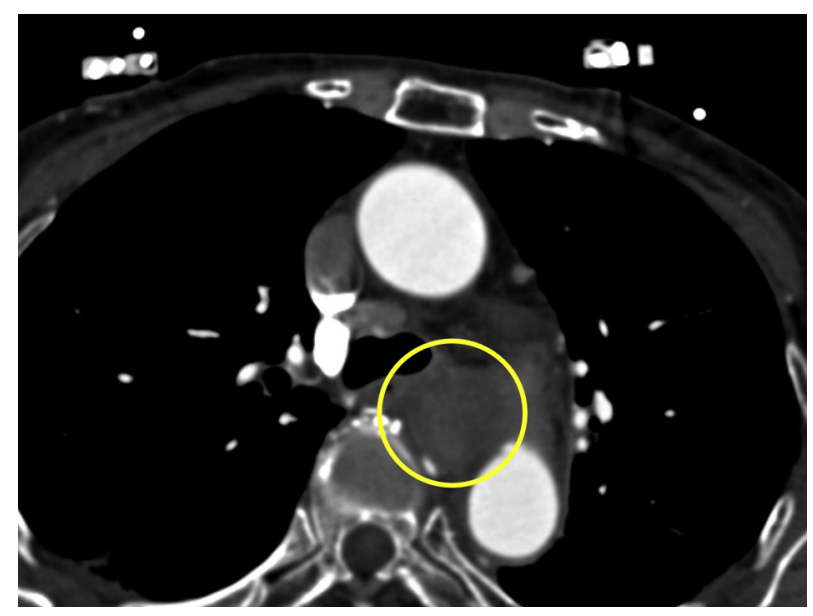

VIDEO 4. The CT after embolization showed that blood flow to the bronchial artery had disappeared. Video available at: https://www.jtcvs.org/ article/S2666-2507(20)30294-7/fulltext.

to be distinguished from a tumor, ${ }^{4}$ but no cases have been useful in distinguishing between aortic dissection and aortic aneurysms as in this case. ${ }^{5}$ In cases that are difficult to discriminate via $\mathrm{CT}$, it is considered that MRI can help to diagnose the relationship with nearby organs such as the aorta.

Regarding treatment indications, BAAs are often treated even when they are asymptomatic because they can be life-threatening at the time of rupture regardless of size. ${ }^{5}$ Treatment methods include surgery and endovascular treatment. In this case, we chose BAE because the patient was elderly and hemodynamically stable.

\section{CONCLUSIONS}

It is necessary to make an accurate diagnosis because aortic dissection or aneurysm and aneurysm from a branch vessel differ in treatment. This case suggests that MRI may be useful for making a diagnosis if there is difficulty even with the use of contrast CT.

\section{References}

1. Fujita J, Akashi K, Kunikane H, Nakajima I, Saito S, Abe S, et al. A case of bronchial artery aneurysm demonstrating a mass shadow on chest X-ray film. Nihon Kyobu Shikkan Gakkai Zasshi. 1991;29:1591-5.

2. Hall RJ, Miller GA, Kerr IH. Ruptured bronchial artery aneurysm mimicking aortic dissection. Br Heart J. 1997;39:909-10.

3. Bak SH, Han H. Diagnosis of bronchial artery aneurysm by computed tomography: a case report. Radiol Case Rep. 2017;12:455-9.

4. Fukunaga A, Okushiba S, Ohno K, Kitashiro S, Kawarada Y, Shitinohe T, et al. Mediastinal bronchial artery aneurysm with hematemesis. Dis Esophagus. 2003; 16:328-31.

5. San Norberto EM, Urbano Garcia J, Montes JM, Vaquero C. Endovascular treatment of bronchial aneurysms. J Thorac Cardiovasc Surg. 2018;156:109-17. 